\title{
Histopathologic features from preoperative biopsies to predict spread through air spaces in early-stage lung adenocarcinoma: a retrospective study
}

\author{
Lanqing Cao, Meng Jia, Ping-Li Sun ${ }^{*}$ and Hongwen Gao
}

\begin{abstract}
Background: Although spread through air spaces (STAS) is a robust biomarker in surgically resected lung cancer, its application to biopsies is challenging. Moreover, limited resection is not an effective treatment for STAS-positive lung adenocarcinoma. This study aimed to identify histologic features from preoperative percutaneous transthoracic needle biopsies (PTNBs) to predict STAS status in the subsequently resected specimens, and thus help in selecting the surgical extent.

Methods: Between January 2014 and December 2015, 111 PTNB specimens and subsequent resection specimens from consecutive lung adenocarcinoma patients were retrospectively examined. Histopathologic features of PTNB specimens and presence of STAS in subsequent resection specimens were evaluated and correlations between them were analyzed statistically.

Results: The study participants had a mean age of 59 years (range, 35-81) and included 50 men and 61 women. Thirty-six patients were positive for STAS whereas 75 were negative. The micropapillary/solid histologic subtypes of lung adenocarcinoma (26 of 39; 66.7\%; $P<0.001$ ), necrotic/tumor debris (31 of $42 ; 73.8 \%$; $P<0.001$ ), intratumoral budding (ITB) (20 of 33; 60.6\%; $P<0.001$ ), desmoplasia (35 of 41; 85.4\%; $P<0.001$ ), and grade 3 nuclei (12 of 14; 85.7\%; $P<0.001)$ were more common in STAS-positive tumors. Micropapillary/solid histologic subtype (OR, 1.35; $95 \%$ Cl: 1.06, 1.67), ITB (OR, 1.64; 95\% Cl: 1.09, 2.83), desmoplasia (OR, 1.83; 95\% Cl: 1.36, 3.12), and N stage (N1 stage: $\mathrm{OR}, 1.37 ; 95 \% \mathrm{Cl}: 1.19,1.87$ ) (N2 stage: OR, 1.29; $95 \% \mathrm{Cl}: 1.07,1.73$ ) were independent predictors of STAS.

Conclusions: Micropapillary/solid histologic subtype, ITB, and desmoplasia in preoperative PTNB specimens were independently associated with STAS in the subsequent resection specimens. Therefore, these can predict STAS and may help to optimize therapeutic planning.
\end{abstract}

Keywords: Histopathologic features, Lung adenocarcinoma, PTNB, Risk stratification, Spread through air spaces

\section{Background}

Lung adenocarcinomas (LAC) have a unique pattern of invasion compared to malignancies originating in other organs. Apart from non-lepidic histologic invasion

\footnotetext{
* Correspondence: pinglisun@jlu.edu.cn

Department of Pathology, The Second Hospital of Jilin University, 218

Ziqiang Road, Changchun, Jilin 130041, China
}

subtypes, infiltrating myofibroblastic stroma, lymphovascular invasion, and pleural invasion, tumor spread through air spaces (STAS), a new distinct invasion concept, was recognized as a pattern of tumor spread in LACs [1]. STAS is defined as micropapillary clusters, solid nests, or single cells beyond the edge of the

(c) The Author(s). 2021 Open Access This article is licensed under a Creative Commons Attribution 4.0 International License, which permits use, sharing, adaptation, distribution and reproduction in any medium or format, as long as you give appropriate credit to the original author(s) and the source, provide a link to the Creative Commons licence, and indicate if changes were made. The images or other third party material in this article are included in the article's Creative Commons licence, unless indicated otherwise in a credit line to the material. If material is not included in the article's Creative Commons licence and your intended use is not permitted by statutory regulation or exceeds the permitted use, you will need to obtain permission directly from the copyright holder. To view a copy of this licence, visit http://creativecommons.org/licenses/by/4.0/ The Creative Commons Public Domain Dedication waiver (http://creativecommons.org/publicdomain/zero/1.0/) applies to the data made available in this article, unless otherwise stated in a credit line to the data. 
primary tumor spreading into the air spaces of the surrounding lung parenchyma [1].

STAS is correlated with a considerable reduction in the recurrence-free survival (RFS) and overall survival (OS) in LACs. The presence of STAS has been associated with more aggressive features and poor prognosis in several histological variations of lung cancer. Furthermore, STAS has been recognized as an exclusion criterion for the diagnosis of adenocarcinoma in situ and minimally invasive adenocarcinoma (MIA) [1]. Specifically, STAS is a robust predictor for the local recurrence of early-stage LACs treated with limited resection $[2,3]$. Since STAS status can be determined only after the operation to date, it cannot offer substantial assistance for operative decisions. No reliable standard assessment system has been reported for evaluating STAS status by frozen tissue sections during surgical procedures [4]. Thus, determining the potential ability to predict STAS status from preoperative biopsy studies can optimize therapeutic planning for LACs.

In this study, the histologic features of percutaneous transthoracic needle biopsy (PTNB) of LACs were reviewed, and we further correlated the histologic findings of the PTNB specimens with those of the corresponding resected tissues. The main goal of this study was to identify the histologic features that can predict tumor behavior in lung biopsy specimens, thus providing clues for optimal surgical treatment planning.

\section{Methods \\ Patients}

This retrospective study was approved by the Ethics Committee of the Second Hospital of Jilin University (Jilin, China). The requirement for informed consent was waived due to the retrospective nature of the study. Between January 2014 and December 2015, 111 consecutive patients underwent PTNB at our hospital, and were pathologically diagnosed with lung adenocarcinoma. Figure 1 shows patients inclusion and exclusion criteria for the study. All patients underwent a subsequent curative surgical resection. Surgical extent was subclassified as segmentectomy, lobectomy, or pneumonectomy; segmentectomies were collectively referred to as limited resection. Patients were excluded from this study if the pathological findings were inconclusive, or if they had a history of a previous lung operation, neoadjuvant therapy, and specific variants of adenocarcinoma such as invasive mucinous adenocarcinoma, fetal or enteric adenocarcinoma, or other specific accompanying components such as squamous, neuroendocrine, or poor differentiation. Clinical parameters, such as patient age, sex, tumor location, and postoperative outcomes, were collected from the medical records.

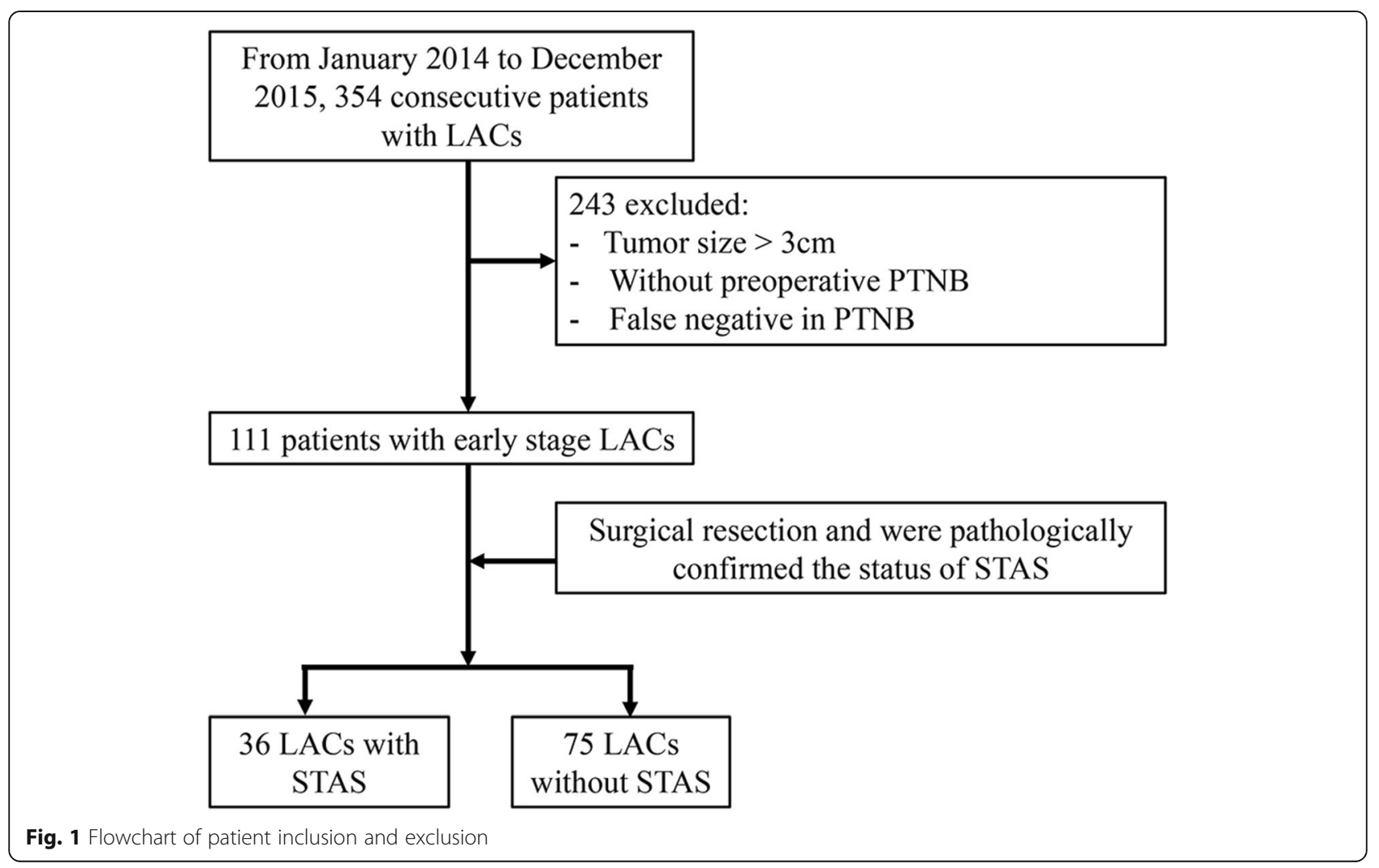




\section{Evaluation of histologic features}

Pathological examination of the PTNB and surgical specimens was performed using sequential 3-mmthick sections stained with hematoxylin and eosin (H\&E). Microscopy and imaging were performed using the Olympus BX51 (batch number: 1E39433; filter model: ND6+, ND25+, LBD+, and OP-), Olympus DP26. And the images were acquired by the acquisition software ACDSee Photo Manager 12 at resolution: $2448 \times 1920$. All tumors were classified according to the latest World Health Organization definitions [1]. All pathological findings were independently evaluated by two single-blinded pathologists. Furthermore, all histological features of PTNB specimens were evaluated by two pathologists blinded to the pathological diagnosis status of subsequent surgical specimens. By definition, STAS was identified as only isolated tumor cells separated from the primary tumor mass and with no direct connection. The mimics and artificial fragments were carefully evaluated and excluded. The following histologic features of PTNB specimens were reviewed: histologic subtype (micropapillary or/and solid vs. others; micropapillary included classical pattern and filigree pattern [5]), intratumoral budding (ITB), necrotic/tumor debris, nuclear grade, desmoplasia, mucin production (extracellular mucin), intracellular mucin, lymphovascular invasion (LVI), and inflammatory reaction. In the subsequent resection specimens, we further evaluated the tumor size and lymph node status. ITB was defined as isolated single cells or a cell cluster composed of $<5$ cells. Intraluminal necrotic/tumor debris was defined as tumor cell apoptosis, ghost tumor cells, and neutrophils. Nuclear grade was categorized as 1 (low), 2 (intermediate), or 3 (high). Grade 1 nuclear features resembled the features seen in adenocarcinoma in situ. Grade 3 nuclear features included nuclear enlargement and pleomorphism, and nucleolar prominence. Nuclear grade 2 was defined as nuclear atypia between grade 3 and grade 1 nuclei. The findings were recorded separately.

\section{Statistical analysis}

Categorical and continuous variables representing the clinical and pathologic findings from the positive and negative STAS groups were compared using the Fisher exact test and Mann-Whitney U test. Multivariable logistic regression analyses were conducted to identify the top independent predictors of STAS. Variables with a $p$ value $<0.10$ from the univariate analyses were included in the multivariable analyses. All statistical analyses were performed using SPSS statistical software, version 21.0 (SPSS Inc., Chicago, IL, USA).

\section{Results}

\section{Demographic and Clinicopathologic findings from resection specimens}

The demographic and clinicopathologic features of our final study population are shown in Table 1, and an example of LAC with STAS is shown in Fig. 2A. The study population had a mean age of 59 years (range, 35-81), and it included 50 men and 61 women. Limited resection was performed in $12.6 \%$ (14 of 111) of patients; fewer patients who were positive for STAS (0 of 14;0\%) underwent limited resection than patients who were negative for STAS (36 of 97; 37.1\%) $(P=0.004)$.

The surgical margin was negative in all patients. Among the 111 resection specimens, the main invasive pattern was acinar in 55 (49.5\%), papillary in six (5.4\%), lepidic in 11 (9.9\%), solid in 23 (20.7\%), and micropapillary in $16(14.5 \%)$ cases. STAS was more frequently detected in tumors with aggressive pathological characteristic, such as higher $\mathrm{N}$ stage $(P=0.004)$, micropapillary/solid histologic subtypes $(P=0.001)$, and LVI $(P=0.032)$.

\section{Correlation between STAS and histologic features of PTNB specimens}

We first evaluated the association between STAS present in the subsequent resection specimens and suspected STAS present in the corresponding PTNB specimens. Only six cases of PTNB with suspected STAS were observed, and no significant correlation with STAS status was found in subsequent surgical specimens. The histologic features of 111 PTNB specimens are summarized in Table 2. A significant relationship was found between the STAS status and the micropapillary/solid histologic subtypes of LAC (26 of 39; 66.7\%; $P<0.001$ ) (Fig. 2B and $\mathbf{C}$ ), necrotic/tumor debris (31 of $42 ; 73.8 \%$; $P<0.001$ ) (Fig. 2D), ITB (13 of 33; 39.4\%; $P<0.001$ ) (Fig. 3A), desmoplasia (35 of 41; 85.4\%; $P<0.001$ ) (Fig. $3 B$ ), and grade 3 nuclei (12 of 14; 85.7\%; $P<0.001$ ) (Fig. 3C). No relationship was found between STAS status and LVI (9 of 19, 47.4\%; $P=0.127$ ) (Fig. 3D), mucinous features $(P=0.859)$, or chronic inflammation $(P=0.835)$ (Table 2).

\section{Histologic predictors of STAS in lung adenocarcinomas}

Histologic subtypes (micropapillary/solid vs. others), along with other features with a $P<0.10$ in univariable analyses, including $\mathrm{N}$ stage, necrotic/tumor debris, desmoplasia, grade 3 nuclei, and adenocarcinoma in situ, were included in a multivariable logistic regression analysis (Table 3). Micropapillary/solid histologic subtype (OR, 1.35; 95\% CI: 1.06, 1.67), ITB (OR, 1.64; 95\% CI: $1.09,2.83$ ), desmoplasia (OR, 1.83; 95\% CI: 1.36, 3.12), and $\mathrm{N}$ stage (N1 stage: OR, 1.37; 95\% CI: 1.19, 1.87) (N2 stage: OR, 1.29; $95 \%$ CI: $1.07,1.73)$ were found to be the 
Table 1 Demographics of 111 patients with lung adenocarcinoma who underwent surgical resection

\begin{tabular}{|c|c|c|c|c|c|}
\hline variable & & $\begin{array}{l}\text { All patients }(n= \\
\text { 111) }\end{array}$ & $\begin{array}{l}\text { Negative for STAS }(n= \\
75)\end{array}$ & $\begin{array}{l}\text { Positive for STAS ( } n= \\
\text { 36) }\end{array}$ & $\begin{array}{l}P \\
\text { value }\end{array}$ \\
\hline Age (years)* & & $59.1 \pm 9.1[35-81]$ & $59.8 \pm 9.2[35-77]$ & $57.6 \pm 8.7[37-81]$ & 0.279 \\
\hline \multirow[t]{2}{*}{ Sex } & Female & 61 & 39 (52.0\%) & $22(61.1 \%)$ & 0.419 \\
\hline & Male & 50 & $36(48.0 \%)$ & 14 (38.9\%) & \\
\hline \multirow[t]{2}{*}{ Smoking status } & Never & 71 & $46(61.3 \%)$ & 25 (69.4\%) & 0.527 \\
\hline & Former or current & 40 & $29(38.7 \%)$ & 11 (30.1\%) & \\
\hline \multirow[t]{2}{*}{ Location } & Upper \& middle lobe & 61 & $38(50.7 \%)$ & $23(63.9 \%)$ & 0.225 \\
\hline & Lower lobe & 50 & 37 (49.3\%) & $13(36.1 \%)$ & \\
\hline \multirow[t]{2}{*}{ Surgery } & $\begin{array}{l}\text { Lobectomy or } \\
\text { pneumonectomy }\end{array}$ & 97 & $61(81.3 \%)$ & 36 (100\%) & 0.004 \\
\hline & Sublobar resection & 14 & $14(18.7 \%)$ & $0(0.0 \%)$ & \\
\hline Tumor Size $(\mathrm{mm})^{*}$ & & $22.7 \pm 7.9[8.1-29.5]$ & $22.3 \pm 8.4[8.1-29.5]$ & $23.4 \pm 6.6[8.3-29.1]$ & 0.446 \\
\hline \multirow[t]{3}{*}{ Lymph Node Status } & No & 89 & $65(86.7 \%)$ & $24(66.7 \%)$ & 0.039 \\
\hline & N1 & 14 & 7 (9.3\%) & $7(19.4 \%)$ & \\
\hline & N2 & 8 & $3(4.0 \%)$ & $5(13.9 \%)$ & \\
\hline \multirow[t]{5}{*}{ Histologic Subtypes } & Lepidic & 11 & $11(14.7 \%)$ & $0(0.0 \%)$ & 0.001 \\
\hline & Acinar & 55 & $46(61.3 \%)$ & $9(25.0 \%)$ & \\
\hline & Papillary & 6 & $2(2.6 \%)$ & $4(11.1 \%)$ & \\
\hline & Micropapillary & 16 & $5(6.7 \%)$ & $11(30.6 \%)$ & \\
\hline & Solid & 23 & $11(14.7 \%)$ & $12(33.3 \%)$ & \\
\hline \multirow{2}{*}{$\begin{array}{l}\text { Lymphovascular } \\
\text { Invasion }\end{array}$} & Absent & 91 & $66(88.0 \%)$ & 25 (69.4\%) & 0.032 \\
\hline & Present & 20 & $9(22.0 \%)$ & $11(30.6 \%)$ & \\
\hline \multirow[t]{2}{*}{ Visceral Pleural Invasion } & Absent & 101 & 68 (90.7\%) & $33(91.7 \%)$ & 0.863 \\
\hline & Present & 10 & 7 (9.3\%) & $3(8.3 \%)$ & \\
\hline
\end{tabular}

* Data are mean \pm standard deviation. STAS, tumor spread through air spaces

independent determinants of STAS in the multivariable analysis model.

\section{Discussion}

This retrospective study quantitatively and systemically evaluated the usefulness of histologic characteristics to predict the presence of STAS in PTNB specimens. Among the various evaluated histologic features, micropapillary/solid histologic subtype, ITB, and desmoplasia were found to be independent predictors of STAS. To the best of our knowledge, this is the first study to assess the role of histologic features of PTNB specimens in predicting STAS as an adverse prognostic indicator in LAC.

The presence of STAS is a powerful independent predictor for poor survival [6-8]. Furthermore, in earlystage LAC with STAS, sublobar/limited resection correlated with a relatively higher risk of recurrence than lobectomy [2, 9-11]. However, no statistical difference was found between lobectomy and sublobar/limited resection in early-stage LAC patients without STAS [12, 13]. These data suggest that an extra wide surgical resection is needed in limited resection of LACs that are positive for STAS; ideally, limited resection should not be considered even in early-stage tumors if findings indicate STAS. Therefore, accurately predicting whether LAC is positive or negative for STAS could assist surgeons in determining which patients are eligible for limited resection, and those who may need an extra lobectomy or postoperative treatment.

PTNB is an effective method for preoperative diagnosis of peripheral lung cancer. However, due to the limited sampling in PTNB, there is a low diagnostic rate or false negative rate in the routine pathology examination. Our data showed a false negative rate for PTNB of $6.5 \%$ in LACs less than $3 \mathrm{~cm}$, and of only $2.3 \%$ in LACs greater than $3 \mathrm{~cm}$. Therefore, in early-stage lung cancer patients, even if a PTNB is negative, radiology examination results should be combined to determine the subsequent treatment of patients. In our study, the micropapillary/solid histologic subtype was found in $17.3 \%$ of STAS-negative cases and in $72.2 \%$ of STASpositive cases. In other studies as well, positive STAS has been predominantly associated with non-lepidic (micropapillary/solid) subtypes [6-8, 14-16]. The micropapillary and solid histologic subtype is associated with aggressive features and indicates poor prognosis; even in 


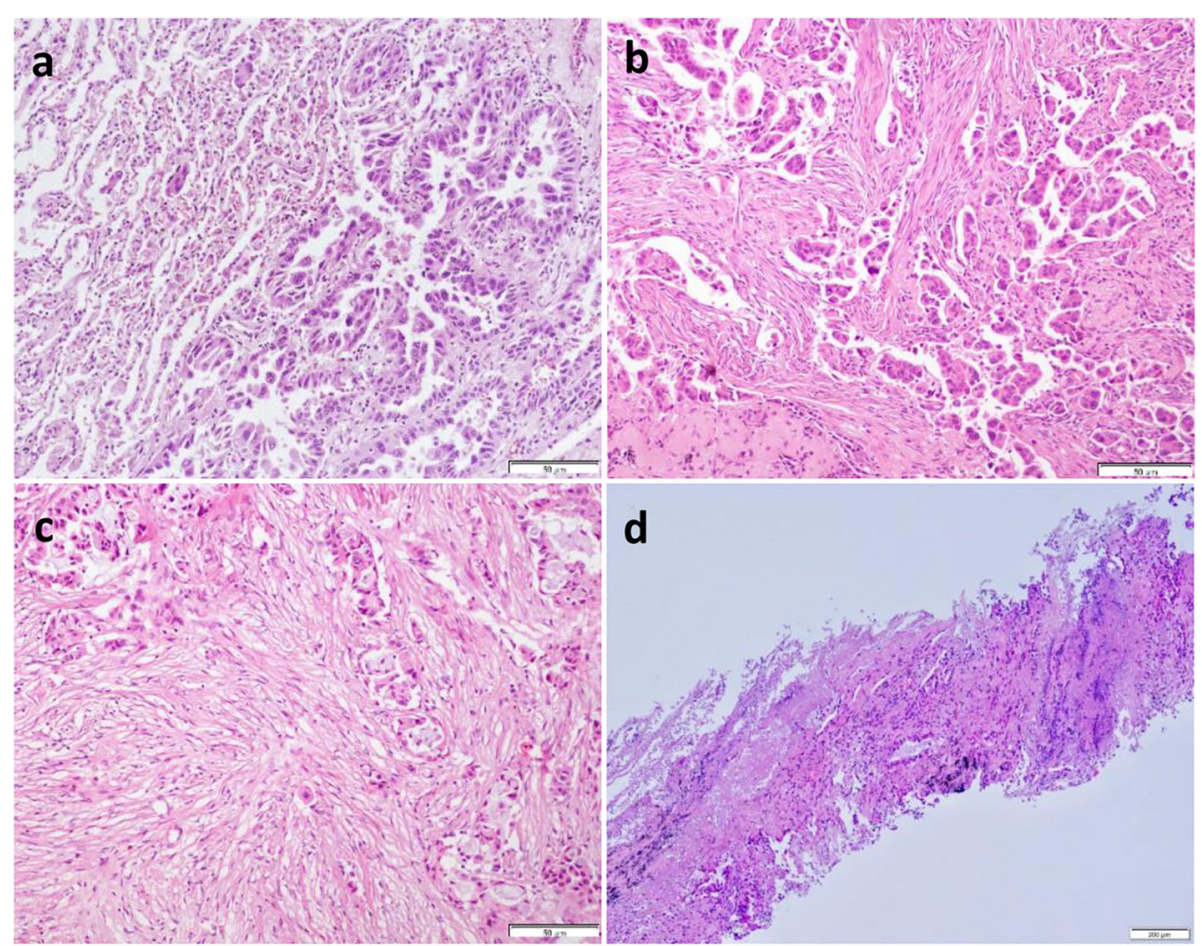

Fig. 2 Histological imaging in patients with tumor spread through air spaces (STAS). (A) Photomicrograph image showing STAS in a lung adenocarcinoma specimen with STAS in surgical specimens. (B and $\mathbf{C}$ ) Photomicrograph images showing micropapillary/solid histologic subtypes of lung adenocarcinoma in PTNB specimens. (D) Photomicrograph image showing necrotic/tumor debris in a lung adenocarcinoma with STAS

Table 2 Association between spread through air spaces and pathologic variables of the biopsy specimens

\begin{tabular}{|c|c|c|c|c|c|}
\hline variable & & All patients & Negative for STAS $(n=75)$ & Positive for STAS $(n=36)$ & $P$ value \\
\hline \multirow[t]{2}{*}{ Histologic subtypes } & Others & 72 & $62(82.7 \%)$ & $10(27.8 \%)$ & $<0.001$ \\
\hline & Micropapillary/Solid & 39 & $13(17.3 \%)$ & $26(72.2 \%)$ & \\
\hline \multirow[t]{2}{*}{ Lymphovascular Invasion } & Absent & 92 & 65 (86.7\%) & $27(75.0 \%)$ & 0.127 \\
\hline & Present & 19 & $10(13.3 \%)$ & $9(25.0 \%)$ & \\
\hline \multirow[t]{2}{*}{ Chronic Inflammation } & Absent & 57 & $38(50.7 \%)$ & $19(52.8 \%)$ & 0.835 \\
\hline & Present & 54 & 37 (49.3\%) & $17(47.2 \%)$ & \\
\hline \multirow[t]{2}{*}{ Mucinous features } & Mucin production & 12 & $7(58.3 \%)$ & $5(62.5 \%)$ & 0.859 \\
\hline & Cytoplasmic mucin & 8 & $5(41.7 \%)$ & $3(37.5 \%)$ & \\
\hline \multirow[t]{2}{*}{ Necrotic/Tumor Debris } & Absent & 69 & $64(85.3 \%)$ & $5(13.9 \%)$ & $<0.001$ \\
\hline & Present & 42 & $11(14.7 \%)$ & $31(86.1 \%)$ & \\
\hline \multirow[t]{2}{*}{ ITB } & Absent & 78 & $62(82.7 \%)$ & $16(44.4 \%)$ & $<0.001$ \\
\hline & Present & 33 & $13(17.3 \%)$ & $20(55.6 \%)$ & \\
\hline \multirow[t]{2}{*}{ Desmoplasia } & Absent & 70 & 69 (92.0\%) & $1(2.8 \%)$ & $<0.001$ \\
\hline & Present & 41 & $6(8.0 \%)$ & 35 (97.2\%) & \\
\hline \multirow[t]{2}{*}{ Grade 3 Nuclei } & Absent & 97 & 73 (97.3\%) & $24(66.7 \%)$ & $<0.001$ \\
\hline & Present & 14 & $2(2.7 \%)$ & 12 (33.3\%) & \\
\hline
\end{tabular}

* Data are mean \pm standard deviation. ITB, intratumoral budding; STAS, tumor spread through air spaces 


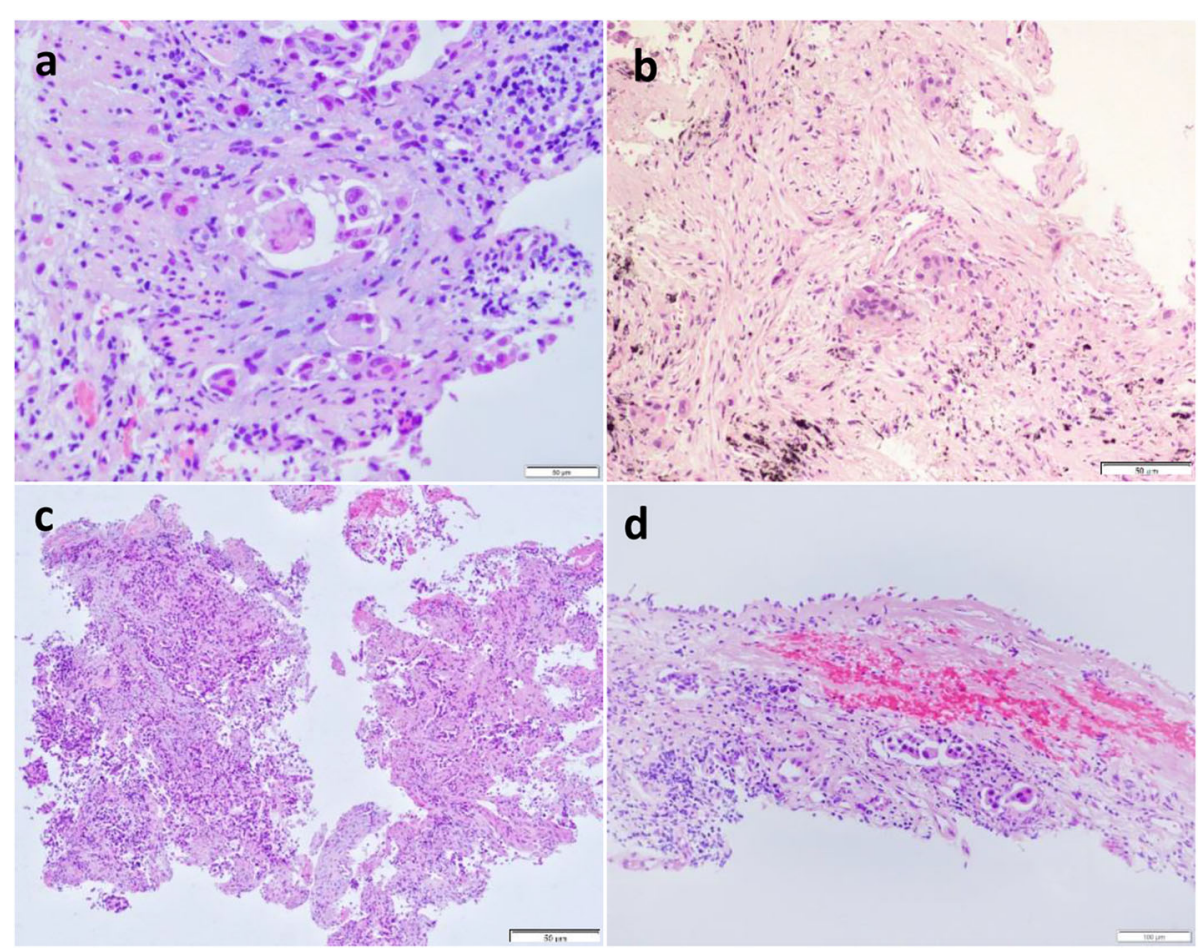

Fig. 3 Histological imaging in patients with tumor spread through air spaces (STAS) in PTNB specimens. (A) Photomicrograph image showing intratumoral budding in a lung adenocarcinoma with STAS. (B) Photomicrograph image showing desmoplasia in a lung adenocarcinoma with STAS. (C) Photomicrograph image showing grade 3 nuclei in a lung adenocarcinoma with STAS. (D) Photomicrograph image showing lymphovascular invasion in a lung adenocarcinoma with STAS. Note: For better reading, the brightness and contrast were modified in (B)

patients with stage IA LCAs, a micropapillary and solid prominent growth pattern indicates worse prognosis [7]. Based on our results, we postulate that the presence of a micropapillary and solid growth pattern in PTNB specimens may be a powerful predictor of STAS. Furthermore, our results suggested that ITB and desmoplasia in PTNB could also predict the presence of STAS, independent of other histologic features, including LVI, necrotic/tumor debris, and grade 3 nuclei.

Tumor budding (TB) is an important independent prognostic factor in colorectal cancer and the routine

Table 3 Multivariable logistic analysis for predictors of STAS

\begin{tabular}{lll}
\hline Variable & Odds Ratio(95\% Cl) & $P$ Value \\
\hline Histologic subtypes & $1.35(1.06-1.67)$ & 0.016 \\
Necrosis/Tumor Debris & $1.03(0.78-1.24)$ & 0.131 \\
ITB & $1.64(1.09-2.83)$ & 0.011 \\
Desmoplasia & $1.83(1.36-3.12)$ & $<0.001$ \\
Grade 3 Nuclei & $1.00(0.41-1.03)$ & 0.464 \\
N stage & 1 & \\
& $1.37(1.19-1.87)$ & 0.013 \\
& $1.29(1.07-1.73)$ & 0.039 \\
\hline
\end{tabular}

$\mathrm{Cl}$, confidence intervals; ITB, intratumoral budding; LVI, lymphovascular invasion reporting of $\mathrm{TB}$ is now advocated using the approach outlined by the International Tumor Budding Consensus Conference guidelines [17]. By definition, STAS was identified as isolated tumor cells separated from the primary tumor mass and with no direct connection to the air spaces; while TB was defined as single tumor cells or small clusters of $\leq 4$ tumor cells at the invasive front stroma. TB has been stratified into peritumoral budding (PTB) and ITB [18]. STAS and TB have special invasion patterns, but it may be difficult to distinguish STAS within air spaces in the alveolar parenchyma beyond the edge of the tumor from TB. However, ITB was located in the tumor center, which could be easily distinguished from STAS; TB was less than 4 cells and almost within the fibrous stroma, while STAS may be beyond 4 cells and primarily within the air spaces. Thus, in most cases, STAS and TB could be clearly distinguished from each another. Tumor dedifferentiation at the invasive fronts shows morphologic features of epithelial-mesenchymal transition (EMT) $[19,20]$ which is a vital underlying molecular mechanism that enhances the tumor cells' ability to survive, invade, and disseminate [19]. The cross-talk between tumor cells and tumor stroma favors tumor progression [21]. Therefore, desmoplasia may affect the tumor microenvironment via various cytokines and growth factors that promote EMT, and subsequently, 
buds and STAS. Supported by our study's results, ITB and desmoplasia in PTNB are associated with a high ratio of STAS in the correspondent resection specimens. In combination with the contraindication for limited resection in LACs with STAS, these predictive factors can optimize surgical decisions regarding adequate extent of surgery or local ablative therapies.

Our study had several limitations. First, since it was a retrospective single-center study with a relatively small sample size, the statistical power was limited. Second, we only evaluated STAS in LCAs, excluding other histological subtypes of lung cancer, such as invasive mucinous adenocarcinomas, squamous cell carcinomas, or adenosquamous cell carcinomas [22-24]. For a better understanding of STAS, more multi-center studies should be conducted including those on other histologic subtypes rather than adenocarcinoma alone. Finally, our study mainly tries to develop a prediction model; however, the generalizability of our results is unclear. Thus, further validation studies are required.

\section{Conclusions}

In conclusion, STAS is a unique tumor invasion pattern of LAC and a strong prognostic factor in LAC, particularly in cases of limited resection. The micropapillary/ solid histologic subtype, ITB, and desmoplasia in PTNB are promising histologic biomarkers for predicting STAS in LACs and may substantially assist the surgeon to optimize the treatment decisions for each patient.

\section{Abbreviations \\ EMT: epithelial-mesenchymal transition; ITB: intratumoral budding; LAC: Lung adenocarcinomas; LVI: Iymphovascular invasion; MIA: minimally invasive adenocarcinoma; OS: overall survival; PTNB: percutaneous transthoracic needle biopsies; RFS: recurrence-free survival; STAS: spread through air spaces}

\section{Acknowledgments}

The authors would like to thank Mr. Xianliang Sha for his technical assistance during the research.

\section{Authors' contributions}

All authors contributed to the study conception and design. Material preparation, data collection, and analysis were performed by LC, MJ and PS. HG contributed to the study as supervisor. The first draft of the manuscript was written by LC, and all authors commented on subsequent versions of the manuscript. All authors have read and approved the final manuscript.

\section{Funding}

This study was supported in major by grants from the Youth Program of the National Natural Science Foundation of China (\#3A5205749429) and in part by grants from the Science and Technology Development Project of Jilin Province (\#3D5197431429 and 3D5204971429).

\section{Availability of data and materials}

The datasets generated or analyzed during the current study are available from the corresponding author upon reasonable request.

\section{Declarations}

Ethics approval and consent to participate

For this retrospective study, institutional review board approval was obtained from the local Ethics Committee of the Second Hospital of Jilin University (Jilin, China). The requirement for informed consent from patients was waived in view of the retrospective nature of the study.

Consent for publication

Not applicable.

\section{Competing interests}

The authors declare that they have no competing interests.

Received: 10 January 2021 Accepted: 20 July 2021

Published online: 11 August 2021

\section{References}

1. Travis WD, Brambilla E, Nicholson AG, Yatabe Y, Austin JHM, Beasley MB, et al. The 2015 World Health Organization classification of lung tumors: impact of genetic, clinical and radiologic advances since the 2004 classification. J Thorac Oncol. 2015;10(9):1243-60. https://doi.org/10.1097/ JTO.0000000000000630.

2. Kadota K, Nitadori J, Sima CS, Ujiie H, Rizk NP, Jones DR, et al. Tumor spread through air spaces is an important pattern of invasion and impacts the frequency and location of recurrences after limited resection for small stage I lung adenocarcinomas. J Thorac Oncol. 2015;10(5):806-14. https://doi.org/1 0.1097/JTO.0000000000000486.

3. Masai K, Sakurai H, Sukeda A, Suzuki S, Asakura K, Nakagawa K, et al. Prognostic impact of margin distance and tumor spread through air spaces in limited resection for primary lung Cancer. Thorac Oncol. 2017;12(12): 1788-97. https://doi.org/10.1016/j.jtho.2017.08.015.

4. Warth A. Spread through air spaces (STAS): a comprehensive update. Transl Lung Cancer Res. 2017;6(5):501-7. https://doi.org/10.21037/tlcr.2017.06.08.

5. Emoto K, Equchi T, Tan KS, Takahashi Y, Aly RG, Rekhtman N, et al. Expansion of the concept of micropapillary adenocarcinoma to include a newly recognized filigree pattern as well as the classical pattern based on 1468 stage I lung adenocarcinomas. J Thorac Oncol. 2019;14(11):1948-61. https://doi.org/10.1016/j.jtho.2019.07.008.

6. Liu Y, Chen D, Qiu X, Duan S, Zhang Y, Li F, et al. Relationship between MTA1 and spread through air space and their joint influence on prognosis of patients with stage I-III lung adenocarcinoma. Lung cancer. 124:211-8.

7. Qiu X, Chen D, Liu Y, Duan S, Zhang F, Zhang Y, et al. Relationship between stromal cells and tumor spread through air spaces in lung adenocarcinoma. Thorac Cancer. 2019;10(2):256-67. https://doi.org/10.1111/1759-7714.12945.

8. Lee JS, Kim EK, Kim M, Shim HS. Genetic and clinicopathologic characteristics of lung adenocarcinoma with tumor spread through air spaces. Lung Cancer. 2018;123:121-6. https://doi.org/10.1016/j.lungcan.2018. 07.020 .

9. Toyokawa G, Yamada Y, Tagawa T, Kozuma Y, Matsubara T, Haratake N, et al. Significance of spread through air spaces in resected pathological stage I lung adenocarcinoma. Ann Thorac Surg. 2018;105(6):1655-63. https://doi. org/10.1016/j.athoracsur.2018.01.037.

10. Bains S, Eguchi T, Warth A, Yeh YC, Nitadori Jl, Woo KM, et al. ProcedureSpecific Risk Prediction for Recurrence in Patients Undergoing Lobectomy or Sublobar Resection for Small i PS adenocarcinoma. Lung cancer 124:211Adenocarcinomas. masbJ Thorac Oncol. 2019;14:72-86.

11. Ren Y, Xie H, Dai C, She Y, Su H, Xie D, et al. Prognostic impact of tumor spread through air spaces in sublobar resection for $1 \mathrm{~A}$ lung adenocarcinoma patients. Ann Surg Oncol. 2019;26(6):1901-18. https://doi. org/10.1245/s10434-019-07296-w.

12. Eguchi T, Kameda K, Lu S, Bott MJ, Tan KS, Montecalvo J, et al. Lobectomy is associated with better outcomes than sublobar resection in spread through air spaces (STAS)-positive T1 lung adenocarcinoma: a propensity scorematched analysis. J Thorac Oncol. 2019;14(1):87-98. https://doi.org/10.1016/j. jtho.2018.09.005.

13. Kadota K, Kushida Y, Kagawa S, Ishikawa R, Ibuki E, Inoue K, et al. Limited resection is associated with a higher risk of Locoregional recurrence than lobectomy in stage I lung adenocarcinoma with tumor spread through air spaces. Am J Surg Pathol. 2019;43(8):1033-41. https://doi.org/10.1097/PAS. 0000000000001285 . 
14. Hu SY, Hsieh MS, Hsu HH, Tsai TM, Chiang XH, Tsou KC, et al. Correlation of tumor spread through air spaces and clinicopathological characteristics in surgically resected lung adenocarcinomas. Lung Cancer. 2018;126:189-93. https://doi.org/10.1016/j.lungcan.2018.11.003.

15. Warth A, Muley T, Kossakowski CA, Goeppert B, Schirmacher P, Dienemann $\mathrm{H}$, et al. Prognostic impact of intra-alveolar tumor spread in pulmonary adenocarcinoma. Am J Surg Pathol. 2015;39(6):793-801. https://doi.org/10.1 097/PAS.0000000000000409.

16. Ding Q, Chen D, Wang X, Wen J, Chen C, Zhang Y, et al. Characterization of lung adenocarcinoma with a cribriform component reveals its association with spread through air spaces and poor outcomes. Lung Cancer. 2019;134: 238-44. https://doi.org/10.1016/j.lungcan.2019.06.027.

17. Lugli A, Kirsch R, Ajioka Y, Bosman F, Cathomas G, Dawson H, et al. Recommendations for reporting tumor budding in colorectal cancer based on the international tumor budding consensus conference (ITBCC) 2016. Mod Pathol. 2017;30(9):1299-311. https://doi.org/10.1038/modpathol.2017.4 6.

18. Lugli A, Vlajnic T, Giger O, Karamitopoulou E, Patsouris ES, Peros G, et al. Intratumoral budding as a potential parameter of tumor progression in mismatch repair-proficient and mismatch repair-deficient colorectal cancer patients. Human Pathol. 2011;42(12):1833-40. https://doi.org/10.1016/j. humpath.2011.02.010.

19. Hanahan D, Weinberg RA. Hallmarks of cancer: the next generation. Cell. 2011;144(5):646-74. https://doi.org/10.1016/j.cell.2011.02.013.

20. Hainaut P, Plymoth A. Targeting the hallmarks of cancer: towards a rational approach to next-generation cancer therapy. Curr Opin Oncol. 2013;25(1): 50-1. https://doi.org/10.1097/CCO.0b013e32835b651e.

21. Kalluri R, Zeisberg M. Fibroblasts in cancer. Nat Rev Cancer. 2016;6:392-401.

22. Tateishi U, Müller NL, Johkoh T, Maeshima A, Asamura H, Satake M, et al. Mucin-producing adenocarcinoma of the lung: thin-section computed tomography findings in 48 patients and their effect on prognosis. J Comput Assist Tomogr. 2005;29(3):361-8. https://doi.org/10.1097/01.rct.0000162820. 08909.e1.

23. Gaeta M, Blandino A, Pergolizzi S, Mazziotti S, Caruso R, Barone M, et al. Patterns of recurrence of bronchioloalveolar cell carcinoma after surgical resection: a radiological, histological, and immunohistochemical study. Lung Cancer. 2003;42(3):319-26. https://doi.org/10.1016/50169-5002(03)00362-3.

24. Liu H, Yin Q, Yang G, Qie P. Prognostic impact of tumor spread through air spaces in non-small cell lung cancers: a Meta-analysis including 3564 patients. Pathol Oncol Res. 2019;25(4):1303-10. https://doi.org/10.1007/s122 53-019-00616-1.

\section{Publisher's Note}

Springer Nature remains neutral with regard to jurisdictional claims in published maps and institutional affiliations.

Ready to submit your research? Choose BMC and benefit from:

- fast, convenient online submission

- thorough peer review by experienced researchers in your field

- rapid publication on acceptance

- support for research data, including large and complex data types

- gold Open Access which fosters wider collaboration and increased citations

- maximum visibility for your research: over $100 \mathrm{M}$ website views per year

At $\mathrm{BMC}$, research is always in progress.

Learn more biomedcentral.com/submissions 